\title{
Hacia un nuevo VIII par: Alternativas de baja complejidad
}

\section{Towards a new vestibular assessment: Low complexity alternatives}

\author{
Pilar Contreras $\mathbf{R}^{1}$, Daniela Toro $\mathbf{N}^{1}$, Gerardo Oberreuter $\mathrm{E}^{1}$, Cristián Barraza $\mathbf{P}^{2}$, Juan Pablo Faúndez $\mathbf{A}^{2}$, \\ Marcia Núñez $\mathrm{T}^{2}$, Maya Kuroiwa $\mathbf{R}^{3}$, Hayo Breinbauer $\mathbf{K}^{3,4}$.
}

\begin{abstract}
RESUMEN
Introducción: El examen funcional del VIII par, mediante la prueba calórica permite conocer la función del canal semicircular horizontal, dejando de lado la evaluación de otras estructuras como el complejo utrículo-sacular.

Objetivo: Evaluar el rendimiento diagnóstico de pruebas vestibulares de bajo costo y complejidad cefálico para suplir esta falencia.

Material y método: En 34 pacientes con indicación de estudio funcional de VIII par se realizaron, además de las pruebas tradicionales, el test visual subjetivo vertical mediante prueba del balde, la prueba de impulso cefálico a ojo desnudo, y la aplicación del cuestionario de sintomatología "Dizziness Handicap Inventory".

Resultados: Considerando el VIII par clásico como patrón de oro, se encuentra evidencia objetiva de patología en 50\% de la muestra. Con la incorporación de los nuevos exámenes esta cifra aumenta a $85 \%$. Se realizó análisis de discrepancia sobre pacientes con resultados normales en el estudio tradicional, pero anormal según los nuevos exámenes. El 100\% de dicho grupo presenta una puntuación patológica en el cuestionario de síntomas. Más aún, los valores de desviación de verticalidad correlacionaron fuertemente con la puntuación de sintomatología ( $r=0,79 ; p=0,002)$.

Conclusiones: El análisis de discrepancia sugiere que los nuevos exámenes son confiables en identificar patología en el estudio. Incluir en el estudio tradicional del VIII par pruebas de baja complejidad y corta duración (menos de 5 minutos en su conjunto) podría aumentar el rendimiento diagnóstico del estudio del equilibrio en hasta 35\%.
\end{abstract}

Palabras clave: Prueba calórica, pruebas funcionales vestibulares, test subjetivo visual vertical, prueba de impulso cefálico.

\footnotetext{
ABSTRACT

Introduction: Traditionally, the assessment of vestibular function is based on the caloric test. This procedure assesses mainly the horizontal semicircular canal function, leaving other vestibular structures aside, such as the utricule-saccule complex.

Aim: To assess the diagnostic performance low complexity tests to compensate for these issues.

Alumno Medicina, Universidad de Chile.

Fonoaudiólogo. Universidad de Chile. Hospital San Juan de Dios.

Médico. Universidad de Chile. Hospital San Juan de Dios.

Médico. Clínica Alemana, Facultad de Medicina Clínica Alemana, Universidad del Desarrollo.
} 
Material and methods: 34 patients with indication for vestibular assessment were recruited. In addition to traditional testing (caloric test and postural provocation maneuvers), the mentioned Subjective Visual Vertical and Head Impulse tests were applied alongside the Dizziness Handicap Inventory for vestibular symptoms.

Results: Considering caloric testing and Dix-Hallpike maneuvers as gold standard, $50 \%$ of the sample presented an objective cause of their symptomatology. When including the new test, this value increases to $85 \%$. A discrepancy analysis was conducted on the group with normal traditional tests and abnormal new tests. $100 \%$ of this group showed symptoms score above pathological levels. Furthermore, there was a strong relationship between deviation on the Visual Vertical test and symptomatology $(r=0,79 ; p=0,002)$.

Conclusion: The discrepancy analysis suggests that the new tests are reliable in determining pathology on this study. It these test are included to traditional testing, diagnostic performance may increase up to $35 \%$.

Key words: Caloric test, vestibular function testing, subjective vertical visual test, head impulse test.

\section{INTRODUCCIÓN}

Este año se cumple un siglo desde que Robert Bárány recibiera el premio Nobel por sus contribuciones a la otoneurología, donde su principal aporte fue desarrollar la prueba calórica ${ }^{1}$. Esta prueba, en conjunto con las maniobras de Dix-Hallpike para determinar vértigo postural paroxístico benigno² constituyen el verdadero núcleo del examen funcional del VIII par craneano $0^{3,4}$. Este estudio ha sido históricamente en nuestro país la principal herramienta y patrón de oro para estudiar el vértigo y los trastornos del equilibrio ${ }^{3-5}$.

Sin embargo la prueba calórica padece importantes limitaciones, al estudiar exclusivamente la función del canal semicircular horizontal ${ }^{6}$. En años recientes han aparecido nuevas alternativas diagnósticas, que intentan salvar esta deficiencia mediante el análisis independiente de la función de cada subestructura del laberinto vestibular.

Intentando sistematizar estos nuevos exámenes (Figura 1) podemos dividir la función vestibular en dos subsistemas: la función angular de canales semicirculares y la función otolítica del complejo utrículo-sacular (debemos además recordar que la función del equilibrio integrada con los sistemas visual y propioceptivo puede ser evaluada mediante la posturografía ${ }^{6,7}$.

Dentro de la función angular de los canales semicirculares es donde ha adquirido gran relevancia la prueba de impulso cefálico asistida por video (VHIT, por sus siglas en inglés "Video Head Impulse Test"), ya que permite medir el reflejo vestíbulo-ocular en todos sus ejes con gran objetividad y precisión, complementando el estudio del canal horizontal en movimiento de alta aceleración angular (la prueba calórica sólo evalúa estímulo de baja aceleración, lejos del
Figura 1. Exámenes para el estudio del equilibrio. Se presenta un esquema anátomo-funcional del equilibrio y qué examen evalúa cada estructura, separándolos en alta y baja complejidad.

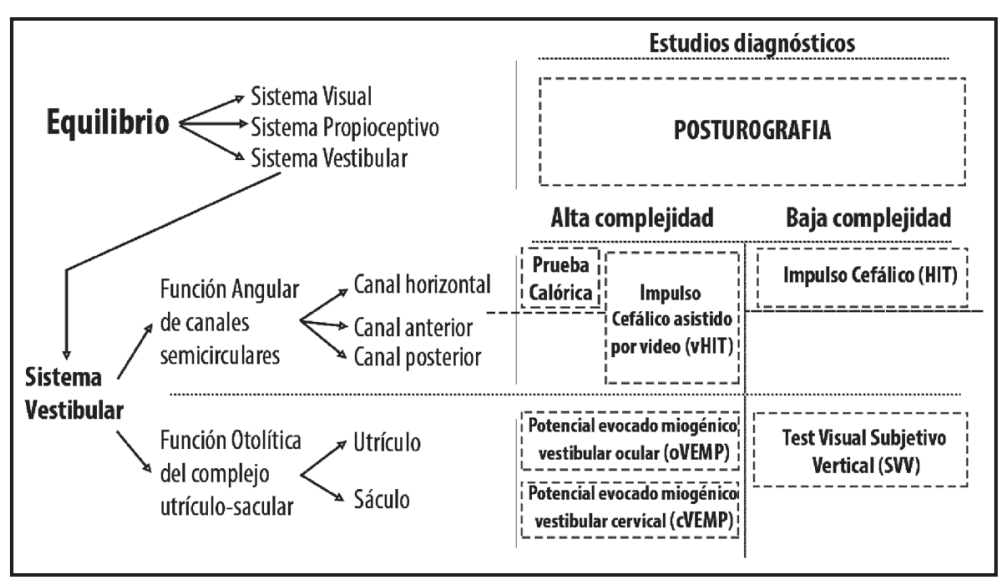


rango fisiológico de movimientos) y especialmente de los canales verticales (anterior y posterior) ${ }^{7-16}$.

Por otro lado, la función de la función otolítica 0 de aceleración lineal ha podido ser objetivada con gran detalle mediante los potenciales evocados miogénicos vestibulares oculares (oVEMP en sus siglas en inglés "Ocular Vestibular Evoked Myogenic Potentials") para la función del utrículo) y cervicales (cVEMP, "cervical VEMP") para la función del sáculo, $0^{6,17-20}$.

Sin embargo estos exámenes requieren del apoyo de tecnología y dispositivos diagnósticos de alta complejidad y de cierto costo económico, los que de momento no se encuentran disponibles en todos los centros donde se realizan estudios del equilibrio. Frente a esta realidad es importante resaltar la existencia de alternativas de baja complejidad para la evaluación de cada una de estas funciones.

En el caso de la función angular, la prueba de impulso cefálico a ojo desnudo (HIT, por sus siglas originales en inglés "Head Impulse Test") tiene un alto valor diagnóstico incluso sin apoyo de video-oculografía ${ }^{7,9}$. Este examen no requiere de ningún material adicional $y$ es posible realizarlo en escasos segundos por un examinador entrenado, teniendo una altísima especificidad con respecto a la prueba calórica pero además logrando identificar algunos casos de alteración vestibular de alta aceleración donde la prueba calórica clásica puede arrojar resultados normales ${ }^{21-25}$. Sin embargo este examen a ojo desnudo es útil solamente para la evaluación del canal horizontal ${ }^{7}$. Por motivos de extensión del artículo, sugerimos revisar sus detalles en esta misma revista en las referencias 7 a 9 .

Asimismo, para la función otolítica, existe una alternativa de bajo costo y breve aplicación en el test visual subjetivo vertical (SVV por sus siglas en inglés) ${ }^{26-29}$. Este estudio consiste en la evaluación de la percepción de verticalidad del paciente. Existen distintas formas de llevarla a cabo. Una de las más simples, más difundida y con mayor validez es la "prueba del balde" (Figura $2)^{27,28}$. En ésta se utiliza un balde que en su interior tiene una línea llamativa en su fondo, mientras que por fuera cuenta con un peso y un transportador que permita medir el grado de desviación de esta línea del eje de verticalidad real dado por la fuerza de gravedad.

En la realización del examen, se solicita al paciente situarse de forma que solo pueda ver el interior del balde, sin claves adicionales en su exterior para orientarse en términos de verticalidad (como paredes u otros objetos). El examinador comienza con el balde rotado en uno u otro sentido (se sugiere repetir la prueba al menos dos veces, una en cada dirección y promediar el resultado). A continuación el examinador rota lentamente el balde y le solicita al paciente indicaciones para llevarlo hasta el punto donde considere que la línea en el interior esté "realmente vertical". Se sugiere repetir la prueba al menos dos veces, cada una comenzando con el balde desviado en sentidos opuestos, y promediar el resultado. En un paciente normal el resultado tendrá ninguna o poca diferencia con la verdadera verticalidad según la gravedad $\left(0^{\circ}\right.$ de diferencia). Mientras que uno con una disfunción del complejo utrículo-sacular (se postula que principalmente el utrículo), se equivocará por más de $2,5^{\circ}$ (límite de normalidad aceptado en la mayoría de las series). Esta desviación ocurrirá hacia el lado lesionado 27,28.

Estudios recientes han intentado demostrar el rendimiento diagnóstico de cada una de estas pruebas como elemento único de análisis en el paciente con patología vestibular, ya sea como método de tamizaje 0 como propuesta para nuevo patrón de or ${ }^{21,24,28,30-33}$. Sin embargo cada test por separado presenta una baja sensibilidad.

Esto es razonable si consideramos que cada prueba evalúa apenas un subsistema del aparato

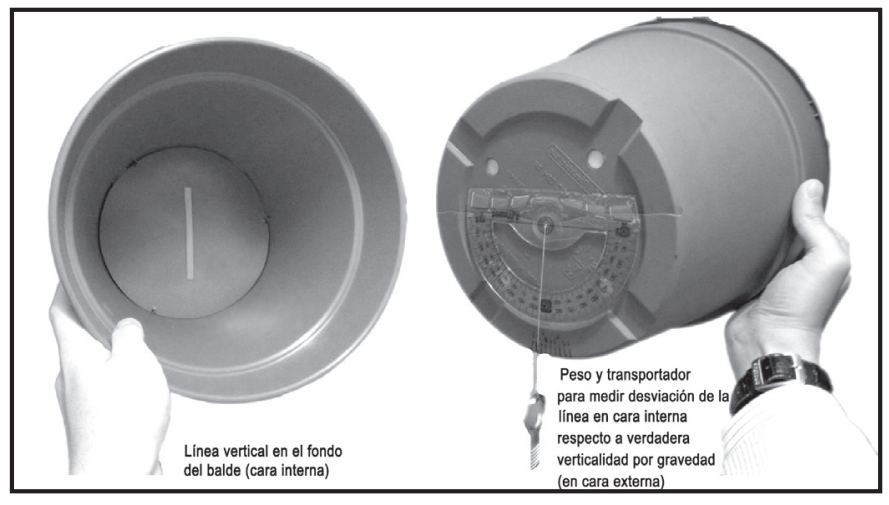

Figura 2. Prueba del balde para el test visual subjetivo vertical. 
vestibular ${ }^{6}$, donde la verdadera utilidad de estos exámenes reside en el desarrollo de una batería mínima de estudios que permitan una evaluación sensible y específica de todas sus funciones.

Dichos estudios se encuentran en curso para los exámenes de mayor complejidad (VHIT y VEMP), con auspiciosos reportes preliminares en las últimas conferencias. Sin embargo no existen en la literatura estudios que aborden la utilidad de las pruebas de baja complejidad (HIT y SVV) como parte de una batería diagnóstica en pacientes con trastornos del equilibrio.

\section{OBJETIVO}

El propósito de este estudio es explorar el rendimiento diagnóstico de HIT y SVV como alternativa de baja complejidad, o apoyo al estudio funcional del VIII par, como primer paso a la propuesta de modificaciones para el estudio del equilibrio que incorpore los avances recientes en otoneurología.

\section{MATERIAL Y MÉTODO}

Estudio exploratorio de rendimiento diagnóstico, prospectivo, con ciego simple. Realizado durante el período de junio a julio de 2014 en el Servicio de Otorrinolaringología del Hospital San Juan de Dios.

La muestra consideró a todos los pacientes consecutivos que tuviesen indicación de realización ambulatoria de un estudio funcional de VIII par por parte de un médico del servicio.

Como criterios de exclusión se consideraron cualquier impedimento que impidiese realizar la totalidad de las pruebas diagnósticas o la autoaplicación de los cuestionarios utilizados, incluyendo por supuesto el deseo de no participar.

Previo consentimiento informado, se realizó en cada paciente el estudio funcional de VIII par de forma habitual (evaluación de equilibrio estático y dinámico, pruebas cerebelosas, evaluación de nistagmo espontáneo, junto con maniobras de Dix-Hallpike y prueba calórica con aire y apoyada por video-oculonistagmografía). Luego un segundo evaluador (que permaneció ciego respecto al resultado de las pruebas previas) condujo SVV por prueba del balde, y HIT a ojo desnudo.

EI SVV se realizó según la descripción ya realizada en la introducción, promediando la desviación neta en grados $\left(^{\circ}\right)$ en dos ensayos. Se utilizó un balde confeccionado por los autores (Figura 2), siguiendo las indicaciones sugeridas por el Centro Nacional de Estudio del Vértigo de München ${ }^{26,27}$. Se consideró una desviación igual o mayor a 2,5 como patológica.

El HIT se realizó según la técnica descrita en otros artículos de esta misma revista ${ }^{7,9}$, registrando como HIT alterado la presencia de sacadas correctivas durante impulsos para el canal semicircular horizontal.

En el análisis se consideró la capacidad de determinar una alteración patológica por el estudio de VIII par clásico como patrón de oro, vale decir la presencia de nistagmo posicional en las maniobras de Dix-Hallpike, o una asimetría mayor de $20 \%$ en la prueba calórica según la fórmula de Jongkees para la velocidad de la componente lenta.

Finalmente se solicitó a los participantes completar el cuestionario de autoaplicación para el Inventario de Discapacidad por Mareo y Vértigo en su versión validada al español (DHI por sus siglas en inglés "Dizziness Handicap Index") ${ }^{34-36}$. Siguiendo recomendaciones internacionales se consideró patológico puntuaciones iguales o mayores a 10.

El DHI se aplicó con el fin de resolver discrepancias entre exámenes según nivel de síntomas en los pacientes, bajo la noción de si uno de los nuevos exámenes (SVV o HIT) propuestos sugería un diagnóstico en un paciente altamente sintomático, aun cuando el patrón de oro imperante resultase negativo (Prueba Calórica o Dix-Hallpike). Es razonable pensar que el nuevo examen resultó ser más sensible que el patrón de oro para encontrar patología.

Se aplicaron análisis de sensibilidad y especificidad en base a los nuevos exámenes (SVV y HIT) considerando el VIII par tradicional como patrón de oro, y el puntaje de DHI como discriminante para los casos de falsos positivos y determinar si en realidad podrían catalogarse como verdaderos positivos. El análisis estadístico se realizó con apoyo de SPSS 20.0.

\section{RESULTADOS}

La muestra reclutada consistió en 34 pacientes, con un promedio de edad de 50,5 años (entre 12 y 80 años, con una desviación estándar de 16,2 años). El 59\% fueron de género femenino.

La realización tanto de las pruebas tradicionales como las nuevas se condujo sin incidentes y 
Tabla 1. Resultados de cada prueba aplicada

\begin{tabular}{|c|c|c|c|c|c|}
\hline \multirow[t]{2}{*}{ Prueba } & \multicolumn{2}{|c|}{ Resultado } & Valor & Sensibilidad & Especificidad \\
\hline & \multicolumn{2}{|c|}{$\begin{array}{cc}\text { Recuento (porcentaje) } \\
\text { Patológico } & \text { Normal }\end{array}$} & $\begin{array}{l}\text { Promedio (DE = Desviación } \\
\text { estándar, Mínimo y Máximo) }\end{array}$ & \multicolumn{2}{|c|}{$\begin{array}{l}\text { (utilizando VIII par tradicional } \\
\text { como patrón de oro) }\end{array}$} \\
\hline Prueba calórica & $\begin{array}{c}15 \\
(44 \%)\end{array}$ & $\begin{array}{c}19 \\
(56 \%)\end{array}$ & $\begin{array}{l}17 \% \text { asimetría (DE } 25 \%, \\
\text { Min 0\%, Max } 85 \% \text { y parálisis } \\
\text { vestibular) }\end{array}$ & $100 \%$ & $100 \%$ \\
\hline Dix-Hallpike & $\begin{array}{c}3 \\
(8,8 \%)\end{array}$ & $\begin{array}{c}31 \\
(91,2 \%)\end{array}$ & $\begin{array}{l}\text { Sin evaluación cuantitativa } \\
\text { posible }\end{array}$ & $18 \%$ & $100 \%$ \\
\hline SVV & $\begin{array}{c}19 \\
(56 \%)\end{array}$ & $\begin{array}{c}15 \\
(44 \%)\end{array}$ & $\begin{array}{c}2,6^{\circ} \\
\left(\mathrm{DE} 2,7^{\circ} ; \operatorname{Min} 0^{\circ} ; \operatorname{Max} 10^{\circ}\right)\end{array}$ & $41 \%$ & $29 \%$ \\
\hline HIT & $\begin{array}{c}6 \\
(17,6 \%)\end{array}$ & $\begin{array}{c}28 \\
(82,4 \%)\end{array}$ & $\begin{array}{l}\text { Sin evaluación cuantitativa } \\
\text { posible }\end{array}$ & $18 \%$ & $82 \%$ \\
\hline $\begin{array}{l}\text { DHI } \\
\text { (corte en } 10 \mathrm{pts} \text { ) }\end{array}$ & $\begin{array}{c}26 \\
(85,3 \%)\end{array}$ & $\begin{array}{c}8 \\
(14,7 \%)\end{array}$ & $\begin{array}{c}\text { 25,3 puntos } \\
\text { (DE 12,4; } \operatorname{Min} 3 ; \operatorname{Max} 54)\end{array}$ & $82 \%$ & $12 \%$ \\
\hline
\end{tabular}

sin efectos adversos. Cabe mencionar que la realización de SVV por prueba del balde y HIT tomó en conjunto menos de 5 minutos en cada caso.

La Tabla 1 resume los hallazgos de la prueba calórica, las maniobras de Dix-Hallpike, SVV, HIT y $\mathrm{DHI}$, incluyendo los valores de sensibilidad y especificidad considerando el examen de VIII par tradicional (prueba calórica y maniobra de DixHallpike) como patrón de oro en la determinación de la presencia de patología vestibular.

Como se aprecia en la Tabla 1, los valores de sensibilidad y especificidad de los nuevos exámenes por separado son sino derechamente pobres, al menos discretos. Se procedió entonces a considerar su aporte no como pruebas independientes sino en conjunto a otras como batería complementaria de exámenes. En la Tabla 2 se presenta un cruce entre la probabilidad de encontrar patología por el VIII par clásico (prueba calórica + Dix-Hallpike) como patrón de oro comparado con el análisis conjunto del VIII par adicionando SVV y HIT, como prueba índice a evaluar.

Ya que la prueba índice incluye al patrón de oro no es sorpresa encontrar una sensibilidad de $100 \%$. Sin embargo destacan el grupo marcado con $\left({ }^{*}\right)$ en la tabla de 12 pacientes (35\% de la muestra) que representan los "falsos positivos", o sea aquellos examinados donde el patrón de oro encontró normalidad y el SVV o el HIT detectó patología. La pregunta reside en determinar si esta discrepancia es efectivamente un falso positivo, 0 una situación donde los nuevos exámenes son más sensibles que el VIII par clásico.

Este análisis (conducente a la determinación de un nuevo patrón de oro) puede manejarse desde varias estrategias estadísticas, siendo la más reco-

Tabla 2. Rendimiento diagnóstico del VIII par en conjunto a los "nuevos exámenes"

\begin{tabular}{|c|c|c|c|c|}
\hline & & \multicolumn{2}{|c|}{$\begin{array}{c}\text { VIII par clásico } \\
\text { (Prueba calórica + Dix-Hallpike) }\end{array}$} & \multirow{4}{*}{$\begin{array}{l}\text { Total } \\
29 \\
5\end{array}$} \\
\hline & & Alterado & Normal & \\
\hline \multirow[t]{4}{*}{ VIII Par + HIT + SVV } & Alterado & 17 & $12^{*}$ & \\
\hline & Normal & 0 & 5 & \\
\hline & & 17 & 17 & \\
\hline & & $\begin{array}{l}\text { Sensibilidad } \\
100 \%\end{array}$ & $\begin{array}{l}\text { Especificidad } \\
29 \%\end{array}$ & \\
\hline
\end{tabular}

Se presentan los niveles de Sensibilidad y Especificidad considerando el VIII par clásico como patrón de oro. Con ( $\left.{ }^{\star}\right)$ se marca el grupo de los falsos positivos: aquellos pacientes considerados normales por el patrón de oro pero evidenciados como patológicos por SVV o por HIT. 
mendada el análisis de sensibilidad y especificidad a submuestras aleatorias y la correlación de éstas con el análisis de la muestra completa.

Este análisis es de momento poco recomendable en este estudio dado el bajo tamaño muestral, por lo que la estrategia de "análisis de discrepancia" es recomendada ${ }^{37}$. Esta consiste en someter al grupo discrepante (el falso positivo) a una tercera prueba que oriente hacia patología o normalidad. En este caso se procedió a correlacionar los hallazgos de SVV y HIT con el DHI para resolver esta discrepancia, lo que se presenta como resumen en la Figura 3.

Destaca que en todos los casos el puntaje en el DHI fue mayor a 10 puntos, considerado el corte para la presencia de discapacidad por mareo o vértigo. Esto sugiere que estos casos revisten "verdaderos positivos".

Para reforzar este concepto en términos del valor diagnóstico de la prueba del balde se realizó prueba de correlación de Pearson entre los valores de SVV y DHI, encontrando un $r=0,786 ; p=0,002$. Esto sugiere una relación fuerte y significativa entre el nivel de desviación en la prueba del balde y la presencia de síntomas en el paciente.

En el caso de HIT, llama la atención que aunque se encontró en apenas 6 pacientes, 3 de ellos fueron en pacientes donde el resultado de la prueba calórica fue normal (siendo que ambos exámenes evalúan la misma función). Buscando nuevamente reforzar la discriminación de HIT como examen diagnóstico se condujo una prueba t de student para comparar las medias de valor de DHI entre el grupo con HIT patológico y HIT normal (en el universo de todos los pacientes con presencia de patología por cualquiera de los exámenes), encontrando significativamente $(p=0,04)$ mayor sintomatología en presencia de sacadas correctivas (media en DHI de 30,2 pts) que en pacientes con esta prueba normal (DHI promedio de 24,1 pts).

$\mathrm{Si}$ consideramos entonces el conjunto de la prueba calórica, Dix-Hallpike, SVV y HIT como patrón de oro (considerando como patológico un resultado alterado en cualquiera de los cuatro), el examen clásico de VIII par presentaría una sensibilidad de $58 \%$ y una especificidad de $100 \%$.

En otros términos, el rendimiento diagnóstico de la evaluación vestibular aumentaría en 35\%, al incorporar estos nuevos exámenes, es decir es capaz de objetivar la presencia de patología en 35\% más de los pacientes.

\section{CONCLUSIONES}

El avance en estudios diagnósticos en otoneurología en la última década nos obliga a revisar la función vestibular en todos sus subsistemas. El estudio del VIII par clásico realizado en nuestro país analiza apenas una parte de la función del canal semicircular lateral, y los fenómenos de canalolitiasis y cupulolitiasis propios del vértigo postural paroxístico benigno. Bajo esta mirada es habitual encontrarse con gran número de pacientes que padecen de algún tipo de trastorno del equilibrio en quienes el examen resulta normal.

En la opinión de los autores esto ocurre simplemente porque tradicionalmente no se evalúan otros componentes del sistema vestibular, principalmente el complejo utrículo-sacular. Así, no es sorprendente
Figura 3. Análisis de discrepancia. Se muestran las puntuaciones en el DHI para los distintos niveles de desviación en el SVV y para los tres casos de HIT alterado dentro del grupo de "falsos positivos" comentados en la Tabla 2. Destaca que en todos los casos el puntaje en el $\mathrm{DHI}$ fue mayor a 10 puntos (representado en el gráfico como la línea continua horizontal), considerado el corte para la presencia de discapacidad por mareo 0 vértigo. Esto sugiere que estos casos revisten "verdaderos positivos".

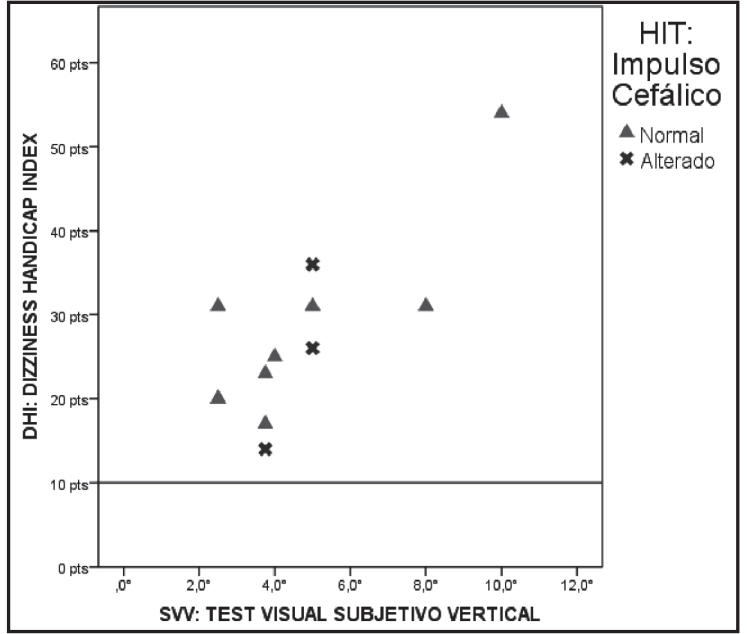


que al incorporar este órgano al estudio, incluso mediante una prueba sencilla como es el SVV mediante uso de un balde como el mostrado en la Figura 2, se aumente el rendimiento diagnóstico del estudio vestibular en más de un tercio. Simplemente se está evaluando una función no considerada previamente.

No sería de extrañar además que la sintomatología del grupo de pacientes exclusivamente positivo a la evaluación por SVV presente principalmente desequilibrio e inestabilidad dado un presumible daño predominante en el complejo utrículo-sacular. Teóricamente los canales semicirculares podrían no estar tan afectados explicando la ausencia de vértigo como sensación de giro dentro de la anamnesis.

Aunque en esta casuística HIT no encontró ningún nuevo paciente que SVV no hubiese encontrado como patológico (Figura 3), llama la atención la detección de alteración de la función del canal horizontal en casos con simetría a la prueba calórica.

Este fenómeno sugiere que los exámenes de alta complejidad tanto para canales semicirculares (vHIT) como para el complejo utrículo-sacular (VEMP) pueden aumentar muchísimo más el rendimiento diagnóstico global del estudio vestibular, contribuyendo estos últimos exámenes además con muchísima información adicional útil para el manejo de patologías específicas.

Un aspecto que de todas formas debe considerarse es que el SVV permanece siendo un examen subjetivo (semejante en esta arista a la audiometría), dependiente de la capacidad y voluntad del paciente de transmitir al examinador su percepción. Esta característica del examen reviste el riesgo de la simulación de la patología.

Aun así los hallazgos en este estudio, indican que la incorporación de dos sencillas pruebas, con un aumento de la duración total del examen no superior a 5 minutos, podría incrementar en más de un tercio el rendimiento diagnóstico de la evaluación del equilibrio.

Además, esta información ayuda a identificar qué subsistemas del equilibrio está afectada (en este caso diferenciar si el componente utrículosacular esta disfuncional), lo que permitiría dirigir y planificar una rehabilitación vestibular específica y personalizada a este paciente. Por ejemplo un paciente sin evidencia de daño en su función angular, pero con alteraciones en su función otolítica no se beneficiaría poco de ejercicios de movimientos oculares y mucho de entrenamiento postural.
Es sin duda necesario comprobar el rendimiento diagnóstico de estas nuevas pruebas bajo una muestra mayor que permita análisis estadísticos más rigurosos. Otra línea necesaria de investigación es cruzar estas pruebas de baja complejidad con los más validados y complejos VHIT y VEMP. La información recabada de la realización de todas estas pruebas en su conjunto, podrían conducir a la determinación de la mínima batería de exámenes vestibulares (en versiones de bajo y alto costo 0 complejidad), o quizás el algoritmo diagnóstico más valido, confiable, sensible y específico para un adecuado estudio integral del vértigo y los trastornos del equilibrio.

\section{REFERENCIAS}

1. Baloh R, Halmagy G, Zee D. The history and future of neuro-otology. Continuum (Minneap Minn) 2012; 18: 1001-15.

2. Vilirie E, Purcell I, Baloh R. The Dix-Hallpike test and the canalith repositioning maneuver. Laryngoscope 2005; 115: 184-7.

3. Riesco-Mac-Clure J, Velasco R. Otoneurología. In: Alonso J, Tato J, eds. Tratado de Otorrinolaringología y Broncoesofagología. Madrid: Editorial Paz Montalvo, 1976; 495-528.

4. Morales C. Otoneurología Clínica. Santiago de Chile: Editorial Universitaria, 1992.

5. PeÑA A. El examen vestibular abreviado, descripción, interpretación y análisis. Rev Otorrinolaringol Cir Cabeza Cuello 2011; 71: 135-44.

6. CuRthoys I. The interpretation of clinical tests of peripheral vestibular function. Laryngoscope 2012; 122: $1342-52$

7. Breinbauer H, Aracena K, Anabalón J, Aladro S, Baeza M. Evaluación de la función vestibular angular: Prueba de impulso cefálico multiaxial a ojo desnudo. Rev Otorrinolaringol Cir Cabeza Cuello 2013; 73: 17-24.

8. Breinbauer $\mathrm{H}$, Anabalón J, Aracena K, Nazal D, Baeza M. Experiencia en el uso del video-Impulso Cefálico (VHIT) en la evaluación del reflejo vestíbulo-ocular para el canal semicircular horizontal. Rev Otorrinolaringol Cir Cabeza Cuello 2013; 73: 115-24.

9. Breinbauer H, Anabalón J. Prueba de impulso cefálico. Rev Otorrinolaringol Cir Cabeza Cuello 2011; 71: 123-30.

10. Mahringer A, Rambold H. Caloric test and videohead-impulse: a study of vertigo/dizziness patients 
in a community hospital. Eur Arch Otorhinolaryngol 2013: Epub ahead of print.

11. Blödow A, Pannasch S, Walther L. Detection of isolated covert saccades with the video head impulse test in peripheral vestibular disorders. Aurus Nasus Larynx 2012; 11: Epub ahead of print.

12. Barti K, Lehnen N, Kohlbecher $S$ y c. Head impulse testing using video-oculography. Ann N Y Acad Sci 2009; 1164: 331-3.

13. Newman-Toker D, Saber A, Mantokoudis G, et al. Quantitative Video-Oculography to Help Diagnose Stroke in Acute Vertigo and Dizziness - Toward an ECG for the Eyes. Stroke 2013; 44: Epub ahead of print.

14. MacDougall H, Weber K, LA M y c. The video head impulse test: diagnostic accuracy in peripheral vestibulopathy. Neurology 2009; 73: 1134-41.

15. Halmagy G, Curthoys I. A clinical sign of canal paresis. Arch Neurol 1988; 45: 737-9.

16. Weber K, MacDougall H, Halmagy G y c. Impulsive testing of semicircular-canal function using videooculography. Ann N Y Acad Sci 2009; 1164: 486-91.

17. Halmagy G, CuRthoys I. Clinical testing of otolith function. Ann N Y Acad Sci 1999; 871: 195-204.

18. UCHINO Y, KusHIRO K. Differences between otolithand semicircular canal-activated neural circuitry in the vestibular system. Nerosci Res 2011; 71: 315-27.

19. Brantberg K. Vestibular evoked myogenic potentials (VEMPs): usefulness in clinical neurotology. Semin Neurol 2009; 29: 541-7.

20. Rosengren S, Welgampola M, Clobatch J. Vestibular evoked myogenic potentials: past, present and future. Clin Neurophysiol 2010; 121: 636-51.

21. Halmagy G, Curthoys I, Cremer P, Henderson C, M S. Head impulse after unilateral vestibular deafferentation validate Ewald's second law. J Vestib Res 1990; 1: 187-97.

22. Pérez N, Rama-López J. Head-Impulse and Caloric Tests in Patients with Dizziness. Otol Neuroto/2003; 24: 913-17.

23. Weber K, Aw S, Todd M y c. Horizontal impulse test detects gentamicin vestibulotoxicity. Neurology 2009; 72: 1417-24.

24. Tuernström F, Nyström A, Magnusson M. How to Uncover the Covert Saccade During the Head Impulse Test. Otol Neurotol 2012; 33: 1583-5.

25. Ulmer E, Bernard-Demanze L, Lacour M. Statistical study of normal canal deficit variation range. Measuremet using Head impulse Test video system. Eur Ann Otorhinolaryngol Head Neck Dis 2011; 128: 278-82.

26. Strupp M, Brandt T. Vestibular Neuritis. Semin Neurol 2009; 29: 509-19.

27. Zwergal A, Rettinger N, Frenzel C, Dieterich M, BRAndT T, Strupp M. A bucket of static vestibular function. Neurology 2009; 72: 1689-92.

28. Sun D, Zuniga M, Davalos-Bichara M, Carey J, AgrawaL Y. Evaluation of a bedside test of utricular function - the bucket test - in older individuals. Acta Otolaryngol 2014; 134: 382-9.

29. Tesio L, Longo S, Rota V. The subjective visual vertical: validation of a simple test. Int J Rehabil Res 2011; 34: 307-15.

30. Walther L, Blödow A. Ocular Vestibular Evoked Myogenic Potential to Air Conducted Sound Stimulation and Video Head Impulse Test in Acute Vestibular Neuritis. Otol Neurotol 2013: Epub ahead of print.

31. Newman-Toker D, Kattah J, Alvernia J, Wang D. Normal head impulse test differentiates acute cerebellar strokes from vestibular neuritis. Neurology 2008; 70: 2378-85.

32. Davalos-Bichara M, Agrawal Y. Normative results of healthy older adults on sandard clinical vestibular test. Otol Neurotol 2014; 35: 297-300.

33. Hirvonen T, Jutila T, Aalto H. Subjective head vertical test reveals subtle head tilt in unilateral peripheral vestibular loss. Eur Arch Otorhinolaryngol 2011; 268: 1523-6.

34. Jacobson GP, Newman CW. The development of the Dizziness Handicap Inventory. Arch Otolaryngol Head Neck Surg 1990; 116: 424-7.

35. Pérez N, Garmendia I, García-Granero M, Martin E, García-Tapia R. Factor analysis and correlation between Dizziness Handicap Inventory and Dizziness Characteristics and Impact on Quality of Life scales. Acta Otolaryngol Supp/ 2001; 545: 145-54.

36. Caldara B, Asenzo Al, Brusotti Paglia Get al. Adaptación cultural y validación del Dizziness Handicap Inventory, versión Argentina. Acta Otolaringol Esp 2012; 63: 106-14.

37. McAdam AJ. Discrepant analysis: how can we test a test? Journal of Clinical Microbiology 2000; 38 : 2027-9.

Dirección: Pilar Contreras R. 